\title{
Control systems on regular time scales and their differential rings
}

\author{
Zbigniew Bartosiewicz • Ülle Kotta • \\ Ewa Pawłuszewicz • Małgorzata Wyrwas
}

Received: 25 March 2010 / Accepted: 15 March 2011 / Published online: 29 March 2011

(C) The Author(s) 2011. This article is published with open access at Springerlink.com

\begin{abstract}
The paper describes an algebraic construction of the inversive differential ring, associated with a nonlinear control system, defined on a nonhomogeneous but regular time scale. The ring of meromorphic functions in system variables is constructed under the assumption that the system is submersive, and equipped with three operators (delta- and nabla-derivatives, and the forward shift operator) whose properties are studied. The formalism developed unifies the existing theories for continuous- and discrete-time nonlinear systems, and accommodates also the case of non-uniformly sampled systems. Compared with the homogeneous case the main difficulties are noncommutativity of delta (nabla) derivative and shift operators and the fact that the additional time variable $t$ appears in the definition of the differential ring. The latter yields that the new variables of the inversive closure, depending on $t$, have to be chosen to be smooth at each dense point $t$ of the time scale.
\end{abstract}

\footnotetext{
Z. Bartosiewicz $(\varangle) \cdot$ M. Wyrwas

Faculty of Computer Science,

Białystok University of Technology, Wiejska 45a,

15-351 Białystok, Poland

e-mail: z.bartosiewicz@pb.edu.pl

M. Wyrwas

e-mail: m.wyrwas@pb.edu.pl

Ü. Kotta

Institute of Cybernetics at Tallinn University of Technology,

Akadeemia tee 21, 12618 Tallinn, Estonia

e-mail:kotta@cc.ioc.ee

E. Pawłuszewicz

Faculty of Mechanical Engineering,

Białystok University of Technology, Wiejska 45c,

15-351 Białystok, Poland

e-mail: e.pawluszewicz@pb.edu.pl
} 
Keywords Time scale $\cdot$ Nonlinear system $\cdot$ Differential ring $\cdot$ Inversive closure

\section{Introduction}

The most popular treatment for nonlinear control systems is from the viewpoint of differential geometry. An alternative and complementary perspective is an algebraic approach based on the use of either differential algebra [10] or vector spaces of differential one-forms over the suitable differential fields or rings of meromorphic functions [9]. The algebraic approach has proven to be more natural for studying the problems like system inversion, realization and reduction, input-output and transfer equivalence. Mathematically speaking, the approach based on differential one-forms consists of three steps. First, one associates an inversive differential field (ring) to a nonlinear control system. Second, one applies the differential operator to "linearize" the given system, yielding a system description in terms of differential one-forms. Finally, one introduces the polynomial operators acting on differential one-forms.

The respective results for the homogeneous time scale case are given in [4] and are based on using the delta-derivative. The latter is a generalization of both the standard time-derivative and that of the difference operator, allowing to merge the continuousand discrete-time cases into a single general framework. However, in order to treat the nontraditional application areas such as biology [16], economics [2] and medicine, where the system dynamics are defined on time scales that are partly continuous and partly discrete, or to accommodate the non-uniformly sampled systems [12], one needs to work on a nonhomogeneous time scale.

The main focus of this paper is on the first step, i.e., to provide an algebraic construction of the inversive differential ring, associated with the nonlinear control system, defined on a nonhomogeneous, but regular time scale. This paves the way to handle the last two steps. We assume that the control system under consideration is submersive and defined by analytic functions. Note that submersivity assumption is not restrictive since it is a necessary condition for system accessibility, see [13].

The extension from homogeneous to nonhomogeneous but regular is far from being trivial. Compared to the homogeneous case, the delta-derivative and the forward shift operators do not commute anymore, but this difficulty is more of a technical nature, making the computations more complex. The main source of difficulty is that the additional time variable $t$ appears in the definition of the differential ring. The latter requires that the new variables of the inversive closure, depending on $t$, have to be chosen to be smooth at each dense point $t$ of the time scale. The preliminary version of the present paper was presented at European Control Conference in 2009, see [5]. The present improved version differs from it in a few key aspects. First, we provide a new example with an explicit construction of the inversive closure. Second, the backward jump operator and the nabla-derivative were introduced and some of their properties proved. Especially, it is demonstrated that the composition of the nabla-derivative and the forward jump operator equals the delta-derivative. The latter yields that the ring of meromorphic functions in system variables can be equipped with three operators (the forward shift, delta- and nabla-derivatives). 
The paper is organized as follows. Section 2 recalls the basic notions of time scale calculus we use later. In Sect. 3 we construct the differential ring of meromorphic functions associated with the nonlinear control system. In Sect. 4 a module over this ring is constructed spanned by the differential one-forms. Section 5 gives the construction of an inversive closure of the differential ring under consideration as well as an example demonstrating the basic steps of the constructions. Section 6 concludes the paper.

\section{Differential calculus on time scales}

The calculus on time scales was initiated by Aulbach and Hilger [3] in order to create a theory that unifies and extends discrete and continuous analysis. For a general introduction, see [6]. Here we recall only these notions that we need in our paper.

In general, a time scale $\mathbb{T}$ is a non-empty closed subset of the set of real numbers $\mathbb{R}$. This definition includes the continuous time case, $\mathbb{T}=\mathbb{R}$, the discrete time case, $\mathbb{T}=\mathbb{Z}$ and $\mathbb{T}=\overline{q^{\mathbb{Z}}}:=\left\{q^{k} \mid k \in \mathbb{Z}\right\} \cup\{0\}$, for $q>1$. The forward jump operator $\sigma: \mathbb{T} \rightarrow \mathbb{T}$ is defined as $\sigma(t)=\inf \{s \in \mathbb{T}: s>t\}$, while the backward jump operator $\rho(t): \mathbb{T} \rightarrow \mathbb{T}$ is defined as $\rho(t)=\sup \{s \in \mathbb{T}: s<t\}$. In addition, if there exists a finite $\max \mathbb{T}, \sigma(\max \mathbb{T}):=\max \mathbb{T}$ and if there exists a finite $\min \mathbb{T}, \rho(\min \mathbb{T}):=\min \mathbb{T}$. As $\mathbb{T}$ is a closed subset of $\mathbb{R}$, both $\sigma(t) \in \mathbb{T}$ and $\rho(t) \in \mathbb{T}$ when $t \in \mathbb{T}$. Finally, for $t \in \mathbb{T}$, the graininess functions $\mu, v: \mathbb{T} \rightarrow[0, \infty)$ are defined by $\mu(t):=\sigma(t)-t$ and $v(t):=t-\rho(t)$.

The time scale calculus is based on the notions of delta derivative $f^{\Delta}$ and nabla derivative $f^{\nabla}$ of a function $f$ defined on $\mathbb{T}$, which are extensions of the standard timederivative in the continuous-time case. Namely, the delta derivative of $f$ at $t$, denoted by $f^{\Delta}(t)$ (or by $\frac{\Delta}{\Delta t} f(t)$ ), is the real number (provided it exists) with the property that given any $\varepsilon>0$ there is a neighborhood $U=(t-\delta, t+\delta) \cap \mathbb{T}$ (for some $\delta>0$ ) such that

$$
\left|(f(\sigma(t))-f(s))-f^{\Delta}(t)(\sigma(t)-s)\right| \leqslant \varepsilon|\sigma(t)-s|
$$

for all $s \in U$. We say that $f$ is delta differentiable on $\mathbb{T}^{\kappa}$ provided $f^{\Delta}(t)$ exists for all $t \in \mathbb{T}^{\kappa}$, where $\mathbb{T}^{\kappa}$ denotes a truncated set consisting of $\mathbb{T}$ except for a possible left-scattered maximal point. Moreover, if $\mathbb{T}_{\kappa}$ denotes a truncated set consisting of $\mathbb{T}$ except for a possible right-scattered minimal point and $f: \mathbb{T} \rightarrow \mathbb{R}$ and $t \in \mathbb{T}_{\kappa}$, then the nabla derivative of $f$ at $t$, denoted by $f^{\nabla}(t)$ (or by $\frac{\nabla}{\nabla t} f(t)$ ), is the real number (provided it exists) with the property that given any $\varepsilon>0$ there is a neighborhood $U=(t-\delta, t+\delta) \cap \mathbb{T}$ (for some $\delta>0$ ) such that

$$
\left|(f(\rho(t))-f(s))-f^{\nabla}(t)(\rho(t)-s)\right| \leqslant \varepsilon|\rho(t)-s|
$$

for all $s \in U$. Moreover, we say that $f$ is nabla differentiable on $\mathbb{T}_{\kappa}$ provided $f^{\nabla}(t)$ exists for all $t \in \mathbb{T}_{\kappa}$.

Remark 1 If $t \in \mathbb{T} \backslash \mathbb{T}^{\kappa}$, then $f^{\Delta}(t)$ is not uniquely defined, since for such a point $t$, small neighbourhoods $\mathscr{U}$ of $t$ consist only of $t$ and $\sigma(t)=t$. Therefore (1) holds for an 
arbitrary number $f^{\Delta}(t)$. This is a reason why we omit a maximal left-scattered point. Similarly, if $t \in \mathbb{T} \backslash \mathbb{T}_{\kappa}$, then $f^{\nabla}(t)$ is not uniquely defined, since for such a point $t$, small neighbourhoods $\mathscr{U}$ of $t$ consist only of $t$ and $\rho(t)=t$. Therefore (2) holds for an arbitrary number $f^{\nabla}(t)$. This is a reason why we omit a minimal right-scattered point.

Example 1 1. If $\mathbb{T}=\mathbb{R}$, then $f^{\Delta}(t)=f^{\nabla}(t)=f^{\prime}(t)$ and $f$ is both delta and nabla differentiable iff it is differentiable in the ordinary sense.

2. If $\mathbb{T}=h \mathbb{Z}, h>0$, then $f^{\Delta}(t)=\frac{f(t+h)-f(t)}{h}$ and $f^{\nabla}(t)=\frac{f(t)-f(t-h)}{h}$ and they always exist.

3. If $\mathbb{T}=\overline{2^{\mathbb{Z}}}$, then $f^{\Delta}(t)=\frac{f(2 t)-f(t)}{t}$ and $f^{\nabla}(t)=\frac{2}{t} \cdot\left[f(t)-f\left(\frac{t}{2}\right)\right]$ for all $t \in \mathbb{T} \backslash\{0\}$.

If $f$ and $g$ are delta differentiable functions at $t \in \mathbb{T}^{\kappa}$, then for any $t \in \mathbb{T}$ holds

1. if $t \in \mathbb{T}^{\kappa}$, then $f$ has at most one delta-derivative at $t$

2. if $f^{\Delta}$ exists, then $f(\sigma(t))=f(t)+\mu(t) f^{\Delta}(t)$

3. for any constants $a, b$ holds $(a f+b g)^{\Delta}(t)=a f^{\Delta}(t)+b g^{\Delta}(t)$

4. $(f g)^{\Delta}(t)=f^{\Delta}(t) g(\sigma(t))+f(t) g^{\Delta}(t)=f(\sigma(t)) g^{\Delta}+f^{\Delta}(t) g(t)$

5. if $g(t) g(\sigma(t)) \neq 0$, then $\left(\frac{f}{g}\right)^{\Delta}(t)=\frac{f^{\Delta}(t) g(t)-f(t) g^{\Delta}(t)}{g(t) g(\sigma(t))}$

Theorem 1 (Chain Rule) [6] Let $f: \mathbb{R} \rightarrow \mathbb{R}$ be continuously differentiable and suppose $g: \mathbb{T} \rightarrow \mathbb{R}$ is delta differentiable. Then $f \circ g: \mathbb{T} \rightarrow \mathbb{R}$ is delta differentiable and $(f \circ g)^{\Delta}(t)=\left\{\int_{0}^{1} f^{\prime}\left(g(t)+h \mu(t) g^{\Delta}(t)\right) \mathrm{d} h\right\} g^{\Delta}(t)$.

If $f$ and $g$ are nabla differentiable functions at $t \in \mathbb{T}_{\kappa}$, then for any $t \in \mathbb{T}$ holds

1. if $t \in \mathbb{T}_{\kappa}$ then $f$ has at most one nabla-derivative at $t$

2. if $f^{\nabla}$ exists, then $f(\rho(t))=f(t)-v(t) f^{\nabla}(t)$

3. for any constants $a, b$ holds $(a f+b g)^{\nabla}(t)=a f^{\nabla}(t)+b g^{\nabla}(t)$

4. $(f g)^{\nabla}(t)=f^{\nabla}(t) g(\rho(t))+f(t) g^{\nabla}(t)=f(\rho(t)) g^{\nabla}+f^{\nabla}(t) g(t)$

5. if $g(t) g(\rho(t)) \neq 0$, then $\left(\frac{f}{g}\right)^{\nabla}(t)=\frac{f^{\nabla}(t) g(t)-f(t) g^{\nabla}(t)}{g(t) g(\rho(t))}$

There are some relationships between delta- and nabla-derivatives.

Theorem 2 [6] (a) Assume that $f: \mathbb{T} \rightarrow \mathbb{R}$ is delta differentiable on $\mathbb{T}^{\kappa}$. Then $f$ is nabla differentiable at $t$ and

$$
f^{\nabla}(t)=f^{\Delta}(\rho(t))
$$

for $t \in \mathbb{T}_{\kappa}$ such that $\sigma(\rho(t))=t$. Additionally if $f^{\Delta}$ is continuous on $\mathbb{T}^{\kappa}$, then $f$ is nabla differentiable at $t$ and (3) holds for any $t \in \mathbb{T}_{\kappa}$.

(b) Assume that $f: \mathbb{T} \rightarrow \mathbb{R}$ is nabla differentiable on $\mathbb{T}_{\kappa}$. Then $f$ is delta differentiable at $t$ and

$$
f^{\Delta}(t)=f^{\nabla}(\sigma(t))
$$

for $t \in \mathbb{T}^{\kappa}$ such that $\rho(\sigma(t))=t$. If, in addition, $f^{\nabla}$ is continuous on $\mathbb{T}_{\kappa}$, then $f$ is delta differentiable at $t$ and (4) holds for any $t \in \mathbb{T}^{\kappa}$. 
By a homogeneous time scale we mean a time scale with constant graininess functions $\mu$ and $\nu$.

Definition 1 [14] A time scale $\mathbb{T}$ is called regular if the following two conditions are satisfied simultaneously:

(i) $\sigma(\rho(t))=t, \quad$ for all $t \in \mathbb{T}$,

(ii) $\rho(\sigma(t))=t, \quad$ for all $t \in \mathbb{T}$.

From (i) it follows that the operator $\sigma: \mathbb{T} \rightarrow \mathbb{T}$ is "onto" while (ii) implies that $\sigma$ is "one-to-one". Therefore, if $\mathbb{T}$ is regular, then $\sigma$ is invertible and $\rho: \mathbb{T} \rightarrow \mathbb{T}$ is also invertible. Moreover, $\sigma^{-1}=\rho$ and $\rho^{-1}=\sigma$.

Remark 2 Every homogeneous time scale is regular, since in that case $\mu \equiv$ const $=$ $h, \sigma(t)=t+h$ and $\rho(t)=t-h$.

Example 2 Time scales $\mathbb{T}=\mathbb{R}, \mathbb{T}=h \mathbb{Z}, h>0$ are both homogeneous and regular. Time scales $\mathbb{T}=\overline{q^{\mathbb{Z}}}$ and $\mathbb{T}=(-\infty, 0] \cup\left\{\frac{1}{k} \mid k \in \mathbb{N}\right\} \cup\left\{\frac{k}{k+1} \mid k \in \mathbb{N}\right\} \cup[1,2]$ are both regular, but not homogeneous.

Proposition 1 [14] A time scale $\mathbb{T}$ is regular if and only if the following two conditions hold:

(i) $\sigma(\min \mathbb{T})=\min (\mathbb{T})$ and $\rho(\max \mathbb{T})=\max (\mathbb{T})$

(ii) each point of the set $\mathbb{T} \backslash\{\min \mathbb{T}$, $\max \mathbb{T}\}$ is either isolated or two-sided dense.

For $f: \mathbb{T} \rightarrow \mathbb{R}$ define $f^{\Delta \zeta}:=\left(f^{\Delta}\right)^{\zeta}$ and $f^{\zeta \Delta}:=\left(f^{\zeta}\right)^{\Delta}, f^{\nabla \zeta}:=\left(f^{\nabla}\right)^{\zeta}$ and $f^{\zeta \nabla}:=\left(f^{\zeta}\right)^{\nabla}$, where $\zeta \in\{\sigma, \rho\}$.

Proposition 2 [7] Let $f: \mathbb{T} \rightarrow \mathbb{R}$ be a delta differentiable function. Assume that the graininess function $\mu$ and $f^{\Delta}$ are delta differentiable. Then

$$
f^{\sigma \Delta}=\left(1+\mu^{\Delta}\right) f^{\Delta \sigma}
$$

Proposition 3 Let $f: \mathbb{T} \rightarrow \mathbb{R}$ be a nabla differentiable function. Assume that the graininess function $v$ and $f^{\nabla}$ are delta differentiable. Then

$$
f^{\rho \nabla}=\left(1-v^{\nabla}\right) f^{\nabla \rho} \text {. }
$$

Proof Applying the relation $f^{\rho}=f-v f^{\nabla}$ to the function $f$ and $f^{\nabla}$ we get $f^{\rho \nabla}=$ $\left(f-v f^{\nabla}\right)^{\nabla}=f^{\nabla}-v^{\nabla} f^{\nabla}-v f^{\nabla \nabla}+v v^{\nabla} f^{\nabla \nabla}=\left(1-v^{\nabla}\right)\left(f^{\nabla}-v f^{\nabla \nabla}\right)=$ $\left(1-v^{\nabla}\right) f^{\nabla \rho}$.

Remark 3 Let $f$ and $f^{\Delta}$ be delta differentiable functions. Then for homogeneous time scales we have $f^{\sigma \Delta}=f^{\Delta \sigma}$.

Remark 4 Let $f$ and $f^{\nabla}$ be nabla-differentiable functions. Then for homogeneous time scales we have $f^{\rho \nabla}=f^{\nabla \rho}$. 
For a function $f: \mathbb{T} \rightarrow \mathbb{R}$ we can talk about second delta-derivatives $f^{\lceil 2\rceil}:=f^{\Delta \Delta}$ provided that $f^{\Delta}$ is delta-differentiable. Similarly we define higher order delta-derivatives $f^{\lceil n\rceil}$, where $n \geqslant 1$. The second nabla-derivatives are defined as follows $f^{\lfloor 2\rfloor}:=$ $f^{\nabla \nabla}$ provided that $f^{\nabla}$ is nabla-differentiable and higher order nabla-derivatives are denoted by $f^{\lfloor n\rfloor}$, where $n \geqslant 1$. Additionally, let $f^{\lceil i . n\rceil}:=\left(f^{\lceil i\rceil}, \ldots, f^{\lceil n\rceil}\right)$ and $f^{\lfloor i . . n\rfloor}:=\left(f^{\lfloor i\rfloor}, \ldots, f^{\lfloor n\rfloor}\right)$, for $0 \leqslant i \leqslant n$ and $f^{\lceil 0\rceil}=f^{\lfloor 0\rfloor}=f$.

\section{Differential rings associated with control systems on regular time scales}

Let $\mathbb{T}$ be a regular time scale. Consider an analytic system, defined on $\mathbb{T}$ :

$$
x^{\Delta}=f(x, u)
$$

where $x \in \mathbb{R}^{n}$ and $u \in \mathbb{R}^{m}, m \leqslant n$. Assume that for all $t \in \mathbb{T}$ the map $(x, u) \mapsto$ $\widetilde{f}(t, x, u)=x+\mu(t) f(x, u)$ generically, i.e., on an open and dense subset of $\mathbb{R}^{n} \times \mathbb{R}^{m}$, defines a submersion, that is generically

$$
\operatorname{rank} \frac{\partial \widetilde{f}(t, x, u)}{\partial(x, u)}=n
$$

holds. ${ }^{1}$ Note that $\mu$ is the graininess function of $\mathbb{T}$ and it depends on $t$ in the contrast with the homogeneous time scale, where $\mu \equiv$ const. Assume that $\mu^{\lceil k\rceil}, k \geqslant 0$, are delta-differentiable functions (or equivalently $\sigma^{\lceil k\rceil}, k \geqslant 0$, are delta-differentiable). Then the functions $\mu^{\lceil k\rceil}, \sigma^{\lceil k\rceil}, k \geqslant 0$, may depend on $t$ as well. We restrict our consideration to such regular time scales for which the graininess function $\mu$ is infinitely many times delta-differentiable. Then the function $\rho=\sigma^{-1}$ is also infinitely many times delta-differentiable. Assume that at each dense point $t_{0} \in \mathbb{T}$ and for some $\varepsilon>0$ the operator $\rho$ may be extended to the interval $\left(t_{0}-\varepsilon, t_{0}+\varepsilon\right)$ in a smooth way. Both homogeneous time scales, i.e., $\mathbb{T}=\mathbb{R}$ and $\mathbb{T}=h \mathbb{Z}, h \geqslant 0$ and $\mathbb{T}=\overline{q^{\mathbb{Z}}}$ satisfy our assumptions. The following example shows that there are regular time scales, which do not satisfy our assumptions.

Example 3 Let $\mathbb{T}=(-\infty, 0] \cup\left\{\frac{1}{n} \mid n \in \mathbb{N} \wedge n \geqslant 3\right\} \cup\left\{\frac{n}{n+1} \mid n \in \mathbb{N}\right\} \cup[1,2]$. The time scale $\mathbb{T}$ is regular and

$$
\mu(t)= \begin{cases}0, & t \in(-\infty, 0] \cup[1,2] \\ \frac{t^{2}}{1-t}, & t \in\left\{\frac{1}{n} \mid n \in \mathbb{N} \wedge n \geqslant 3\right\} \\ \frac{(t-1)^{2}}{2-t}, & t \in\left\{\frac{n}{n+1} \mid n \in \mathbb{N}\right\}\end{cases}
$$

\footnotetext{
${ }^{1}$ Assumption (6) is not restrictive since it is a necessary condition for system accessibility, see [13].
} 
and

$$
\mu^{\Delta}(t)=\left\{\begin{array}{lll}
0, & t \in(-\infty, 0] \cup[1,2] \\
\frac{1}{1-2 t}-1, & t \in\left\{\frac{1}{n} \mid n \in \mathbb{N} \wedge n \geqslant 3\right\} \\
\frac{1}{3-2 t}-1, & t \in\left\{\frac{n}{n+1} \mid n \in \mathbb{N}\right\} \backslash\left\{\frac{2}{3}\right\} \\
-1, & t=\frac{2}{3}
\end{array} .\right.
$$

Then $\mu$ is delta differentiable but $\mu^{\Delta}$ is not delta-differentiable (it is only continuous), since $\mu^{\lceil 2\rceil}(0)$ and $\mu^{\lceil 2\rceil}(1)$ do not exist.

Moreover the operator $\rho$ of the following form

$$
\rho(t)= \begin{cases}t, & t \in(-\infty, 0] \cup[1,2] \\ \frac{t}{1+t}, & t \in\left\{\frac{1}{n} \mid n \in \mathbb{N} \wedge n \geqslant 3\right\} \\ \frac{1}{2-t}, & t \in\left\{\frac{n}{n+1} \mid n \in \mathbb{N}\right\}\end{cases}
$$

cannot be extended in a smooth way to the intervals $\left(-\varepsilon_{1}, \varepsilon_{1}\right),\left(1-\varepsilon_{2}, 1+\varepsilon_{2}\right)$ containing the dense points 0 and 1 , for any $\varepsilon_{1}, \varepsilon_{2}>0$.

Let us consider the infinite set of (independent) real indeterminates

$$
\mathscr{C}:=\left\{x_{i}, i=1, \ldots, n, u_{j}^{\lceil k\rceil}, j=1, \ldots, m, k \geqslant 0\right\}
$$

and the set

$$
\mathscr{C}:=\mathscr{C} \cup\{t\}
$$

where $t$ is an indeterminate with values in $\mathbb{T}$.

Let $\mathscr{R}$ be the ring of functions that depend on a finite number of variables from the set $\mathscr{C}$ and are meromorphic with respect to $x$ and $u^{\lceil 0 . . k\rceil}$ for the fixed time $t \in \mathbb{T}$, and for the fixed $x$ and $u^{\lceil 0 . . k\rceil}$ each function from $\mathscr{R}$ is infinitely many times deltadifferentiable with respect to $t$. Note that the ring $\mathscr{R}$ is commutative, but it can have zero divisors, so it is impossible to construct the quotient field of $\mathscr{R}$. For instance, if $\mathbb{T}=\mathbb{Z}$, then the following function

$$
\varphi(t)= \begin{cases}1, & \text { if } t=1 \\ 0, & \text { if } t \neq 1\end{cases}
$$

belongs to $\mathscr{R}$ and $\varphi$ is a zero divisor, because for $\psi \in \mathscr{R}$ defined by

$$
\psi(t)= \begin{cases}0, & \text { if } t=1 \\ t, & \text { if } t \neq 1\end{cases}
$$

we get $\varphi \cdot \psi \equiv 0$. 
Note that if we fix the time $t$, then we get the field $\mathscr{R}_{t}$ of functions that are meromorphic with respect to $x$ and $u^{\lceil 0 . . k\rceil}$.

For each $F \in \mathscr{R}$ there is $k \geqslant 0$ such that $F$ depends on $t, x$ and $u^{\lceil 0 . . k\rceil}$. Let $\sigma_{f}: \mathscr{R} \rightarrow \mathscr{R}$ be an operator defined by

$\sigma_{f}(F)\left(t, x, u^{\lceil 0 . . k+1\rceil}\right):=F\left(t+\mu(t), x+\mu(t) f(x, u), u^{\lceil 0 . . k\rceil}+\mu(t) u^{\lceil 1 . . k+1\rceil}\right)$.

Such defined $\sigma_{f}$ is an endomorphism. Since $\mathbb{T}$ is regular and assumption (6) holds, $\sigma_{f}$ is injective. Moreover, if $\mu(t) \equiv 0$, then $\sigma_{f}=\mathrm{id}$.

Now let us define the operator $\Delta_{f}: \mathscr{R} \rightarrow \mathscr{R}$ as follows

$$
\Delta_{f}(F)\left(t, x, u^{\lceil 0 . . k+1\rceil}\right):=\frac{\sigma_{f}(F)\left(t, x, u^{\lceil 0 . . k+1\rceil}\right)-F\left(t, x, u^{\lceil 0 . . k\rceil}\right)}{\mu(t)}
$$

if $\mu(t)>0$, and

$$
\begin{aligned}
\Delta_{f}(F)\left(t, x, u^{\lceil 0 . . k+1\rceil}\right):= & \frac{\partial F}{\partial t}\left(t, x, u^{\lceil 0 . . k\rceil}\right)+\frac{\partial F}{\partial x}\left(t, x, u^{\lceil 0 . . k\rceil}\right) f(x, u) \\
& +\sum_{i=0}^{k} \frac{\partial F}{\partial u^{\lceil i\rceil}}\left(t, x, u^{\lceil 0 . . k\rceil}\right) u^{\lceil i+1\rceil},
\end{aligned}
$$

if $\mu(t)=0$.

Remark 5 Let $F \in \mathscr{R}$. Then by (7) $\sigma_{f}(F)=F+\mu \Delta_{f}(F)$.

By the above remark one can easily show that

$$
\Delta_{f}\left(\sigma_{f}(F)\right)=\left(1+\mu^{\Delta}\right) \sigma_{f}\left(\Delta_{f}(F)\right) .
$$

Therefore, for nonhomogeneous time scales

$$
\Delta_{f} \sigma_{f} \neq \sigma_{f} \Delta_{f}
$$

The map $\Delta_{f}$ defined by (7) for system (5) satisfies a suitable generalization of Leibniz rule (the proof is similar to the proof of Proposition 3.8 in [4]):

$$
\Delta_{f}(F G)=\sigma_{f}(F) \Delta_{f}(G)+\Delta_{f}(F) G
$$

so $\mathscr{R}$ is endowed with a $\sigma_{f}$-derivation and therefore it is a differential ring which can be also called $\sigma_{f}$-differential ring.

Remark 6 For $\mathbb{T}=\mathbb{R}, \sigma_{f}=\mathrm{id}$ and $\Delta_{f}=\frac{\mathrm{d}}{\mathrm{d} t}$ is the total time derivative. Then one gets the differential ring as in [9]. 


\section{Module over the differential ring}

Consider the infinite set of symbols

$$
\mathrm{d} \mathscr{C}=\left\{\mathrm{d} x_{i}, i=1, \ldots, n, \mathrm{~d} u_{j}^{\lceil k\rceil}, j=1, \ldots, m, k \geqslant 0\right\}
$$

and denote by $\mathscr{E}$ the module over the ring $\mathscr{R}$ spanned by the elements of $\mathrm{d} \mathscr{C}$, namely

$$
\mathscr{E}=\operatorname{span}_{\mathscr{R}} \mathrm{d} \mathscr{C}
$$

Any element of $\mathscr{E}$ has the form

$$
\omega=\sum_{i=1}^{n} A_{i} \mathrm{~d} x_{i}+\sum_{k \geqslant 0} \sum_{j=1}^{m} B_{j k} \mathrm{~d} u_{j}^{\lceil k\rceil}
$$

where only a finite number of coefficients $B_{j k}$ are nonzero elements of $\mathscr{R}$. The elements of $\mathscr{E}$ will be called the one-forms.

Let us define the operator $\mathrm{d}: \mathscr{R} \rightarrow \mathscr{E}$ as follows

$$
\mathrm{d} F\left(t, x, u^{\lceil 0 . . k\rceil}\right):=\sum_{i=1}^{n} \frac{\partial F}{\partial x_{i}}\left(t, x, u^{\lceil 0 . . k\rceil}\right) \mathrm{d} x_{i}+\sum_{\ell=0}^{k} \sum_{j=1}^{m} \frac{\partial F}{\partial u_{j}^{\lceil\ell\rceil}}\left(t, x, u^{\lceil 0 . . k\rceil}\right) \mathrm{d} u_{j}^{\lceil\ell\rceil} .
$$

We will say that $\omega \in \mathscr{E}$ is an exact one-form if $\omega=\mathrm{d} F$ for some $F \in \mathscr{R}$. Note that the operator $\mathrm{d}$ is not the standard total differential, since we do not differentiate the function $F$ with respect to $t$. Such definition of d guarantees the commutativity of operators $\mathrm{d}$ and $\sigma_{f}$ and $\mathrm{d}$ and $\Delta_{f}$, what will be shown later.

Let $\omega=\sum_{i} A_{i} \mathrm{~d} \zeta_{i}$ be a one-form, where $A_{i} \in \mathscr{R}$ and $\zeta_{i} \in \mathscr{C}$. We define the operators $\Delta_{f}: \mathscr{E} \rightarrow \mathscr{E}$ and $\sigma_{f}: \mathscr{E} \rightarrow \mathscr{E}$ by

$$
\begin{aligned}
\Delta_{f}(\omega) & :=\sum_{i}\left\{\Delta_{f}\left(A_{i}\right) \mathrm{d} \zeta_{i}+\sigma_{f}\left(A_{i}\right) \mathrm{d}\left[\Delta_{f}\left(\zeta_{i}\right)\right]\right\} \\
\sigma_{f}(\omega) & :=\sum_{i} \sigma_{f}\left(A_{i}\right) \mathrm{d}\left[\sigma_{f}\left(\zeta_{i}\right)\right]
\end{aligned}
$$

where $\Delta_{f}\left(u^{\lceil k\rceil}\right)=u^{\lceil k+1\rceil}, k \geqslant 0$ and the dynamics of the system (5) is used to compute $\Delta_{f}\left(x_{i}\right)$, i.e., $\Delta_{f}\left(x_{i}\right)=f_{i}(x, u)$, where $f=\left(f_{1}, \ldots, f_{n}\right)$. Since $\sigma_{f}\left(A_{i}\right)=$ $A_{i}+\mu(t) \Delta_{f}\left(A_{i}\right)$, then

$$
\Delta_{f}(\omega)=\sum_{i}\left\{\Delta_{f}\left(A_{i}\right) \mathrm{d} \zeta_{i}+\left(A_{i}+\mu(t) \Delta_{f}\left(A_{i}\right)\right) \mathrm{d}\left[\Delta_{f}\left(\zeta_{i}\right)\right]\right\}
$$

Proposition 4 For an arbitrary time scale $\mathbb{T}$ and a function $F \in \mathscr{R}$ we have $\mathrm{d}\left[\Delta_{f}(F)\right]=\Delta_{f}(\mathrm{~d} F)$ and $\mathrm{d}\left[\sigma_{f}(F)\right]=\sigma_{f}(\mathrm{~d} F)$. 
Proof The proof is the same as for the homogeneous time scales, see [4].

Proposition 5 Let $\omega \in \mathscr{E}$. Then $\sigma_{f}(\omega)=\omega+\mu(t) \Delta_{f}(\omega)$.

Proof The proof is the same as for the homogeneous time scale, see [4].

All the results of this section coincide with the corresponding results in [9] in case when $\mathbb{T}=\mathbb{R}, \Delta_{f}=\frac{\mathrm{d}}{\mathrm{d} t}$ and $\sigma_{f}=\mathrm{id}$.

Remark 7 Note that the operator $\Delta_{f}$ is well defined. In particular if $F$ is infinitely many times delta-differentiable with respect to $t$, then $\Delta_{f}(F)$ is also infinitely many times delta-differentiable with respect to $t$.

\section{Inversive closure of $\mathscr{R}$}

We would like to work with the differential ring $\mathscr{R}$ that is inversive, i.e., $\sigma_{f}^{-1}(F)$ is defined for all $F \in \mathscr{R}$. In general, the ring $\mathscr{R}$ is not inversive, thus we will construct an inversive closure $\mathscr{R}^{*}$ of the differential ring $\mathscr{R}$, i.e., a differential overring $\mathscr{R}^{*}$ that is inversive.

We need the preimages of the elements from $\mathscr{R}$ with respect to $\sigma_{f}$. If $\sigma_{f}$ is not surjective, then, for some $F \in \mathscr{R}, \sigma_{f}^{-1}(F)$ may not exist. However, it is always possible to embed $\mathscr{R}$ into its inversive closure $\mathscr{R}^{*}$, see [8]. Then $\sigma_{f}^{-1}(F) \in \mathscr{R}^{*}$, for all $F \in \mathscr{R}$ and $\sigma_{f}$ can be extended to $\mathscr{R}^{*}$ in such a way that $\sigma_{f}: \mathscr{R}^{*} \rightarrow \mathscr{R}^{*}$ becomes an automorphism.

Observe that $\sigma$ is surjective as a map on a regular time scale, but it may not be the case for $\sigma_{f}$.

In contrast to the homogeneous time scale, the additional time variable $t$ appears in $\mathscr{R}$ and we must know its preimage with respect to $\sigma_{f}$. Let $\pi_{t}\left(t, x, u^{\lceil 0 . . k\rceil}\right)=$ $t, \pi_{x}\left(t, x, u^{\lceil 0 . . k\rceil}\right)=x$ and $\pi_{u^{\lceil\ell\rceil}}\left(t, x, u^{\lceil 0 . . k\rceil}\right)=u^{\lceil\ell\rceil}, \ell \geqslant 0$. Then $\pi_{t}, \pi_{x}, \pi_{u}\lceil\ell\rceil \in \mathscr{R}$. Since the considered time scale is regular and $\mu^{\lceil k\rceil}, k \geqslant 0$, are delta-differentiable functions, we have

$$
\sigma_{f}^{-1}\left(\pi_{t}\right)(t)=\rho(t) \quad \text { with } \rho \in \mathscr{R} \text {. }
$$

Moreover

$$
\sigma_{f}^{-1}(\mu)(t)=t-\rho(t)=v(t) \quad \text { with } v \in \mathscr{R} \text {. }
$$

Now we need the preimages with respect to $\sigma_{f}$ of the functions $\pi_{x}$ and $\pi_{u^{\lceil\ell\rceil}}$.

Let us fix the time $t \in \mathbb{T}$. Then $\mathscr{R}_{t}$ is the field of meromorphic functions and $\operatorname{span}_{\mathscr{R}_{t}}\{\mathrm{~d} x, \mathrm{~d} u\}$ is the space of the dimension $n+m$. Let $\mathscr{Z}_{t}$ be a complementary subspace to the space $\operatorname{span}_{\mathscr{R}_{t}}\left\{\mathrm{~d} x_{t}^{\sigma}\right\}$, i.e.,

$$
\operatorname{span}_{\mathscr{R}_{t}}\{\mathrm{~d} x, \mathrm{~d} u\}=\operatorname{span}_{\mathscr{R}_{t}}\left\{\mathrm{~d} x_{t}^{\sigma}\right\} \oplus \mathscr{Z}_{t} .
$$


Since $x_{t}^{\sigma}=\tilde{f}(t, \cdot)=\left(\widetilde{f}_{1}(t, \cdot), \ldots, \widetilde{f}_{n}(t, \cdot)\right), \operatorname{span}_{\mathscr{R}_{t}}\left\{\mathrm{~d} x_{t}^{\sigma}\right\}=\operatorname{span}_{\mathscr{R}_{t}}\left\{\mathrm{~d} \widetilde{f}_{1}, \ldots, \mathrm{d} \widetilde{f}_{n}\right\}$. By assumption (6), we get

$$
\operatorname{dim} \operatorname{span}_{\mathscr{R}_{t}}\left\{\mathrm{~d} x_{t}^{\sigma}\right\}=n
$$

for all $t \in \mathbb{T}$. Then $\operatorname{dim} \mathscr{Z}_{t}=m$ and

$$
\mathscr{Z}_{t}=\operatorname{span}_{\mathscr{R}_{t}}\left\{\mathrm{~d} z_{1}^{t}, \ldots, \mathrm{d} z_{m}^{t}\right\}=: \operatorname{span}_{\mathscr{R}_{t}}\left\{\mathrm{~d} z_{t}\right\}
$$

where $\mathrm{d} z_{k}^{t} \in \operatorname{span}_{\mathscr{R}_{t}}\{\mathrm{~d} x, \mathrm{~d} u\}, k=1, \ldots, m$. Therefore, there exists a vector valued function $z_{t}=\varphi_{t}(x, u) \in \mathbb{R}^{m}$ such that the one-forms $\mathrm{d} z_{t}$ generate the subspace $\mathscr{Z}_{t}$. Note that the vector valued function $\varphi_{t}$ is not unique and can be found in many different ways for an isolated point $t$. For isolated points the above construction basically coincides with that in [1]. However, what complicates the construction of the backward shift in general is that we must choose the vector valued function $\varphi_{t}$ in such a way that it is smooth at each dense point $t$ from the time scale $\mathbb{T}$.

Let us recall that $x^{\sigma}(t, x, u)=\widetilde{f}(t, x, u)=x+\mu(t) f(x, u) \in \mathbb{R}^{n}$, where $f(\cdot)$ describes the dynamics of system (5), and $z_{t}=\varphi_{t}(x, u) \in \mathbb{R}^{m}$ is a function such that $\mathrm{d} z_{t}$ generates complementary subspace $\mathscr{Z}_{t}$ for all $t \in \mathbb{T}$. Condition (8) means that the map $(x, u) \mapsto\left(x_{t}^{\sigma}, z_{t}\right)=\left(\tilde{f}(t, x, u), \varphi_{t}(x, u)\right)$ is a (local) diffeomorphism for all $t \in \mathbb{T}$. This implies that (locally and generically) there exists a vector-valued function $\psi_{t}$ such that

$$
(x, u)=\psi_{t}\left(x_{t}^{\sigma}, z_{t}\right) .
$$

The function $\psi_{t}$ is parameterized by $t \in \mathbb{T}$. Note that the function $\psi_{t}\left(x_{t}^{\sigma}, z_{t}\right)=$ $\psi\left(t, x_{t}^{\sigma}, z_{t}\right)$ is infinitely many times delta-differentiable at arbitrary isolated points from the time scale $\mathbb{T}$. Moreover, since for each dense point $t \in \mathbb{T}$ the function $\rho$ can be extended in a smooth way to the interval $(t-\varepsilon, t+\varepsilon)$, for some $\varepsilon>0$, from the Implicit Function Theorem with Parameters (see [11]), the function $\psi\left(t, x_{t}^{\sigma}, z_{t}\right)$ is infinitely many times delta-differentiable at each dense point $t \in \mathbb{T}$. Therefore $\psi_{t}\left(x_{t}^{\sigma}, z_{t}\right)=\psi\left(t, x_{t}^{\sigma}, z_{t}\right)$ is infinitely many times delta-differentiable at arbitrary point $t \in \mathbb{T}$.

Finally, for the fixed time $t$, let $\mathscr{R}_{t}^{*}$ be a field extension of $\mathscr{R}_{t}$ consisting of functions that are meromorphic with respect to a finite number of the independent variables

$$
\mathscr{C}_{t}^{*}:=\left\{x, u^{\lceil k\rceil}, z_{t}^{\langle-\ell\rangle}, k \geqslant 0, \ell \geqslant 1\right\}
$$

Let $\sigma_{f}^{-1}\left(z_{t}\right)=z_{t}^{\langle-1\rangle}$ and $\sigma_{f}^{-1}\left(z_{t}^{\langle-i+1\rangle}\right):=z_{t}^{\langle-i\rangle}$ if $\mu(t)>0$. Note that for $\mu(t)=0, \sigma_{f}^{-1}=\mathrm{id}$, so each element from the set $\mathscr{C}$ has the preimage and we do not have to add new variables. Thus $\mathscr{C}_{t}^{*}=\mathscr{C}$ if $t \in\{t \in \mathbb{T} \mid \mu(t)=0\}$. Since $\sigma_{f}^{-1}\left(\pi_{t}\right)=\rho$ and

$$
(x, u)=\psi_{t}\left(x_{t}^{\sigma}, z_{t}\right)=\psi_{t}\left(\sigma_{f}(x), \sigma_{f}\left(z_{t}^{\langle-1\rangle}\right)\right)=\sigma_{f}\left(\psi_{t}\left(x, z_{t}^{\langle-1\rangle}\right)\right),
$$


then

$$
\sigma_{f}^{-1}\left(\pi_{x}, \pi_{u}\right)= \begin{cases}\psi_{t}\left(x, z_{t}^{\langle-1\rangle}\right), & \text { if } \mu(t)>0 \\ \left(\pi_{x}, \pi_{u}\right), & \text { if } \mu(t)=0\end{cases}
$$

Therefore there exist functions $\psi_{t}^{s}, s=1, \ldots, n+m$, such that

$$
\begin{gathered}
\sigma_{f}^{-1}\left(\pi_{x_{i}}\right)= \begin{cases}\psi_{t}^{i}\left(x, z_{t}^{\langle-1\rangle}\right), & \text { if } \mu(t)>0 \\
\pi_{x_{i}}, & \text { if } \mu(t)=0\end{cases} \\
\sigma_{f}^{-1}\left(\pi_{u_{j}}\right)= \begin{cases}\psi_{t}^{n+j}\left(x, z_{t}^{\langle-1\rangle}\right), & \text { if } \mu(t)>0 \\
\pi_{u_{j}}, & \text { if } \mu(t)=0\end{cases}
\end{gathered}
$$

and if $\mu(t)>0$, then $\sigma_{f}^{-1}\left(\pi_{u_{j}^{\Delta}}\right)=\frac{1}{v(t)}\left[u_{j}-\sigma_{f}^{-1}\left(\pi_{u_{j}}\right)\right]=\frac{1}{v(t)}\left[u_{j}-\right.$ $\left.\psi_{t}^{n+j}\left(x, z_{t}^{\langle-1\rangle}\right)\right]$, if $\mu(t)=0$, then $\sigma_{f}^{-1}\left(\pi_{u_{j}^{\Delta}}\right)=\pi_{u_{j}^{\Delta}}$. So using the induction principle and $\sigma_{f}^{-1}\left(\pi_{u_{j}^{\lceil i\rceil}}\right)=\frac{1}{v(t)}\left[u_{j}^{\lceil i-1\rceil}-\sigma_{f}^{-1}\left(\pi_{u_{j}^{\lceil i-1\rceil}}\right)\right], i \geqslant 1$, one can show that for $j=1, \ldots, m$ and $k \geqslant 1$ we have $\sigma_{f}^{-1}\left(\pi_{u_{j}^{\lceil k\rceil}}\right)=\sum_{i=0}^{k-1}(-1)^{i}[v(t)]^{-i-1} u_{j}^{\lceil k-i-1\rceil}+$ $(-1)^{k}[v(t)]^{-k} \psi_{t}^{n+j}\left(x, z_{t}^{\langle-1\rangle}\right)$ if only $\mu(t)>0$ and $\sigma_{f}^{-1}\left(\pi_{u_{j}^{\lceil k\rceil}}\right)=\pi_{u_{j}^{\lceil k\rceil}}$ if $\mu(t)=0$. Hence $\sigma_{f}$ can be extended to $\mathscr{R}_{t}^{*}$ and it is an automorphism of $\mathscr{R}_{t}^{*}$. Although the choice of variables $z_{t}=\varphi_{t}(x, u)$ is not unique, each possible choice brings up a field extension of $\mathscr{R}_{t}$ that is isomorphic to $\mathscr{R}_{t}^{*}$.

We need the extension of operator $\Delta_{f}$ to variables $z_{t}^{\langle-\ell\rangle}, \ell \geqslant 1$, in the case of $\mu(t)>0$; it is done by using

$$
\Delta_{f}\left(z_{t}^{\langle-\ell\rangle}\right):=\frac{z_{t}^{\langle-\ell+1\rangle}-z_{t}^{\langle-\ell\rangle}}{\mu(t)} .
$$

The extension of operator $\Delta_{f}$ to $\mathscr{R}_{t}^{*}$ can be made in the analogy to (7). Such operator $\Delta_{f}$ is now a $\sigma_{f}$-derivation of $\mathscr{R}_{t}^{*}$, so $\mathscr{R}_{t}^{*}$ is a differential field.

Now let $\mathscr{R}^{*}$ be a subring of the ring that is the Tikhonov product of the fields $\mathscr{R}_{t}^{*}$ for $t \in \mathbb{T}$, i.e.,

$$
\mathscr{R}^{*} \subseteq \prod_{t \in \mathbb{T}} \mathscr{R}_{t}^{*}=\left\{h: \mathbb{T} \rightarrow \bigcup_{t \in \mathbb{T}} \mathscr{R}_{t}^{*} \mid h(t) \in \mathscr{R}_{t}^{*}\right\}
$$

Let $\mathscr{R}^{*}$ be defined as a ring that contains functions that are infinitely many times delta-differentiable with respect to $t$ for the fixed variables from the following set

$$
\mathscr{C}^{*}:=\left\{x, u^{\lceil k\rceil}, z^{\langle-\ell\rangle}, k \geqslant 0, \ell \geqslant 1\right\},
$$


where $z^{\langle-\ell\rangle}$ is parameterized by $t \in \mathbb{T}$. For the fixed time $t, \mathscr{R}^{*}$ is $\mathscr{R}_{t}^{*}$. Then $\mathscr{R}^{*}$ is a ring extension of the ring $\mathscr{R}$ and both $\sigma_{f}$ and $\Delta_{f}$ are well defined on $\mathscr{R}^{*}$. Moreover, the operator $\Delta_{f}$ is now a $\sigma_{f}$-derivation of $\mathscr{R}^{*}$, so $\mathscr{R}^{*}$ is a differential ring.

Let $\mathbf{w}:=\left(x, u, u^{\lceil 1\rceil}, \ldots, z^{\langle-1\rangle}, z^{\langle-2\rangle}, \ldots\right)$. Instead of variables $x, u^{\lceil k\rceil}, z^{\langle-\ell\rangle}, k \geqslant$ $0, \ell \geqslant 1$ the notation $\mathbf{w}$ will be used. Writing $F(\mathbf{w})$ we mean a function that depends on a finite number of variables from the set $\mathscr{C}^{*}$.

Additionally, the operator $\nabla_{f}: \mathscr{R}^{*} \rightarrow \mathscr{R}^{*}$ can be defined as follows

$$
\nabla_{f}(F)(t, \mathbf{w}):=\frac{F(t, \mathbf{w})-\sigma_{f}^{-1}(F)(t, \mathbf{w})}{v(t)},
$$

if $v(t) \neq 0$, and

$$
\nabla_{f}(F)(t, \mathbf{w}):=\Delta_{f}(F)(t, \mathbf{w})
$$

if $v(t)=0$. Directly from (9) and (10) we get

Proposition 6 Let $F \in \mathscr{R}^{*}$. Then $\sigma_{f}\left(\nabla_{f}(F)\right)=\Delta_{f}(F)$ and $\nabla_{f}(F)=\sigma_{f}^{-1}\left(\Delta_{f}(F)\right)$.

Proof If $\nu(t) \neq 0$ and $F \in \mathscr{R}^{*}$, then $\sigma_{f}\left(\nabla_{f}(F)\right)=\frac{\sigma_{f}(F)-F}{\mu}=\Delta_{f}(F)$. Hence $\nabla_{f}(F)=\sigma_{f}^{-1}\left(\sigma_{f}\left(\nabla_{f}(F)\right)\right)=\sigma_{f}^{-1}\left(\Delta_{f}(F)\right)$. For $v(t)=0$ we have $\sigma_{f}=\mathrm{id}$, so then $\Delta_{f}=\nabla_{f}$ and both conditions are satisfied.

Corollary 1 Note that in general we have

$$
\left.\left.\left.\nabla_{f}^{k}(F)=(\underbrace{\sigma_{f}^{-1}\left(\Delta _ { f } \cdots \left(\sigma _ { f } ^ { - 1 } \left(\Delta_{f}\right.\right.\right.}_{k \text {-times }}(F))\right)\right)\right)=\left(\sigma_{f}^{-1} \circ \Delta_{f}\right)^{k}(F)
$$

By Proposition 6 the images of nabla-derivatives with respect to operator $\sigma_{f}$ equal to the delta-derivatives of some function and consequently belong to the ring $\mathscr{R}^{*}$.

Corollary 2 Note that $\nabla_{f}(F) \in \mathscr{R}^{*}$. Moreover, for $k \geqslant 1, \sigma_{f}^{-1}\left(\Delta_{f}^{k}(F)\right)=$ $\nabla_{f}\left(\Delta_{f}^{k-1}(F)\right)$ and in particular, $\sigma_{f}^{-1}\left(u^{\lceil k\rceil}\right)=\left(u^{\lceil k-1\rceil}\right)^{\nabla}$.

Remark 8 Let $\mathbb{T}$ be a regular time scale. Then for $\varphi: \mathbb{T} \rightarrow \mathbb{R}$ we have $\Delta_{f}(\varphi)(t)=$ $\varphi^{\Delta}(t)$ and $\nabla_{f}(\varphi)(t)=\frac{\varphi(t)-\sigma_{f}^{-1}(\varphi)(t)}{\nu(t)}=\frac{\varphi(t)-\varphi(\rho(t))}{\nu(t)}=\varphi^{\nabla}(t)$. Therefore the operator $\nabla_{f}$ is a generalization of the nabla-derivative defined for functions on the time scale. 
Proposition 7 Let $\mathbb{T}$ be the regular time scale. Then for any function $F \in \mathscr{R}^{*}$ we have

$$
\begin{aligned}
& \nabla_{f}(F)(t, \mathbf{w}) \\
& = \begin{cases}\frac{F(t, \mathbf{w})-\sigma_{f}^{-1}(F)(t, \mathbf{w})}{v(t)}, & \text { if } v(t)>0 \\
\frac{\partial F}{\partial t}(t, \mathbf{w})+\frac{\partial F}{\partial x}(t, \mathbf{w}) f(x, u)+\sum_{i \geqslant 0} \frac{\partial F}{\partial u^{\lceil i\rceil}}(t, \mathbf{w}) u^{\lceil i+1\rceil}, & \text { if } v(t)=0 .\end{cases}
\end{aligned}
$$

Proof For $v(t) \neq 0$ the relation comes from definition (9). If $v(t)=0$, then $\mu(t)=\sigma_{f}(v(t))=\sigma_{f}(0)=0$ and $\Delta_{f}=\nabla_{f}$. Because for arbitrary time scale, if $\mu(t)=0$, then (see Proposition 3.3 in [4]) $\Delta_{f} F(t, \mathbf{w})\left(x, u^{\lceil 0 . . k+1\rceil}\right)=$ $\frac{\partial F}{\partial x}\left(x, u^{\lceil 0 . . k+1\rceil}\right) \cdot f(x, u)+\sum_{k \geqslant 0} \frac{\partial F}{\partial u^{\lceil 0 . k\rceil}}\left(x, u^{\lceil 0 . . k\rceil}\right) \cdot u^{\lceil 1 . . k+1\rceil}$, hence $\nabla_{f}(F)(t, \mathbf{w})=$ $\frac{\partial F}{\partial t}(t, \mathbf{w})+\frac{\partial F}{\partial x}(t, \mathbf{w}) \cdot f(x, u)+\sum_{i \geqslant 0} \frac{\partial F}{\partial u^{\lceil i\rceil}}(t, \mathbf{w}) \cdot u^{\lceil i+1\rceil}$.

Remark 9 Let $F \in \mathscr{R}^{*}$. If $v(t) \neq 0$, then by (9)

$$
\sigma_{f}^{-1}(F)(t, \mathbf{w})=F(t, \mathbf{w})-v(t) \cdot \nabla_{f}(F)(t, \mathbf{w}) .
$$

Obviously for $v(t)=0$, we get $\sigma_{f}^{-1}(F)=F$.

Proposition 8 Let $\mathbb{T}$ be the regular time scale. Then for $F \in \mathscr{R}^{*}$ we have

$$
\left(\nabla_{f} \circ \sigma_{f}^{-1}\right)(F)=\left(1-v^{\nabla}\right) \cdot\left(\sigma_{f}^{-1} \circ \nabla_{f}\right)(F)
$$

Proof Using (11) we get $\nabla_{f}\left(\sigma_{f}^{-1}(F)\right)=\nabla_{f}(F)-\nabla_{f}\left[v \cdot \nabla_{f}(F)\right]=\nabla_{f}(F)-$ $v^{\nabla} \cdot \nabla_{f}(F)-v \cdot \nabla_{f}^{2}(F)+v \cdot v^{\nabla} \cdot \nabla_{f}^{2}(F)=\left(1-v^{\nabla}\right) \cdot\left(\nabla_{f}(F)-v \nabla_{f}^{2}(F)\right)=$ $\left(1-v^{\nabla}\right) \cdot \sigma_{f}^{-1}\left(\nabla_{f}(F)\right)$.

Corollary 3 Note that for nonhomogeneous time scales' operators $\nabla_{f}$ and $\sigma_{f}^{-1}$ do not commute, i.e., $\nabla_{f} \sigma_{f}^{-1} \neq \sigma_{f}^{-1} \nabla_{f}$ and additionally taking the image of (12) with respect to $\sigma_{f}$ we get

$$
\left(\Delta_{f} \circ \sigma_{f}^{-1}\right)(F)=\left(1-v^{\Delta}\right) \cdot \nabla_{f}(F)
$$

The map $\nabla_{f}$ satisfies a suitable generalization of Leibniz rule (the proof is similar to the proof of Proposition 3.8 in [4]):

$$
\nabla_{f}(F G)=\sigma_{f}^{-1}(F) \nabla_{f}(G)+\nabla_{f}(F) G,
$$

so $\mathscr{R}^{*}$ is endowed with a $\sigma_{f}^{-1}$-derivation and therefore it is a differential ring.

Now we study a simple example of a dynamical control system, defined on a regular time scale $\mathbb{T}$ and demonstrate the construction of $\mathscr{R}^{*}$. 
Example 4 Let $\mathbb{T}=\overline{2^{\mathbb{Z}}}$. Then since $\mu(t)=t \not \equiv$ const, $\mathbb{T}$ is nonhomogeneous, but it is regular. Additionally, the operator $\rho(t)=\frac{t}{2}$ can be extended to a neighborhood of the dense point 0 in a smooth way. Consider the nonlinear dynamical system

$$
x^{\Delta}=(x+u)^{2}
$$

defined on $\mathbb{T}$, where $x, u \in \mathbb{R}$. System (13) can be rewritten in the form

$$
x^{\sigma}=x+t(x+u)^{2} \text {. }
$$

Observe that

$$
\operatorname{rank}[1+2 t(x+u), 2 t(x+u)]=1,
$$

for all $t \in \mathbb{T}$. Then $\sigma_{f}$ is an injective endomorphism.

In this case $\mathscr{R}$ is the ring containing functions that are meromorphic with respect to variables $x, u^{\lceil k\rceil}, k \geqslant 0$, and infinitely many times delta-differentiable with respect to $t$. We would like to find the inversive closure $\mathscr{R}^{*}$ of $\mathscr{R}$.

One can choose $z_{t}=u-t(x+u)^{2}$. Then the following map

$$
(x, u) \mapsto\left(x+t(x+u)^{2}, u-t(x+u)^{2}\right)
$$

is a diffeomorphism for all $t \in \mathbb{T}$ and

$$
\left\{\begin{array}{l}
x=x^{\sigma}-t\left(x^{\sigma}+z_{t}\right)^{2} \\
u=z_{t}+t\left(x^{\sigma}+z_{t}\right)^{2} .
\end{array}\right.
$$

Note that

$$
\operatorname{span}_{\mathscr{R}^{*}}\{\mathrm{~d} x, \mathrm{~d} u\}=\operatorname{span}_{\mathscr{R}^{*}}\left\{\mathrm{~d} x^{\sigma}\right\} \oplus \operatorname{span}_{\mathscr{R}^{*}}\left\{\mathrm{~d} z_{t}\right\} .
$$

Let us introduce new variables $z_{t}^{\langle-i\rangle}, i \geqslant 1$. Then

$$
\begin{aligned}
\sigma_{f}^{-1}\left(\pi_{t}\right)(t) & =\frac{t}{2}, \\
\sigma_{f}^{-1}\left(\pi_{z_{t}\langle-i\rangle}\right)\left(z_{t}^{\langle-i-1\rangle}\right) & =z_{t}^{\langle-i-1\rangle}, \quad i \geqslant 1 \\
\sigma_{f}^{-1}\left(\pi_{x}\right)\left(t, x, z_{t}^{\langle-1\rangle}\right) & =x-\frac{t}{2}\left(x+z_{t}^{\langle-1\rangle}\right)^{2} \\
\sigma_{f}^{-1}\left(\pi_{u}\right)\left(t, x, z_{t}^{\langle-1\rangle}\right) & =z_{t}^{\langle-1\rangle}+\frac{t}{2}\left(x+z_{t}^{\langle-1\rangle}\right)^{2},
\end{aligned}
$$

where $z_{0}^{\langle-i\rangle}=z_{0}=u$, for $i \geqslant 1$. Moreover, for $k \geqslant 1$

$$
\sigma_{f}^{-1}\left(\pi_{u^{\lceil k\rceil}}\right)=\left(u^{\lceil k-1\rceil}\right)^{\nabla} \in \mathscr{R}^{*}
$$


so all the variables $t, x, u^{\lceil k\rceil}, k \geqslant 0$ have preimages in $\mathscr{R}^{*}$. Then $\mathscr{R}^{*}$ is a ring extension of $\mathscr{R}$ consisting of functions that are meromorphic with respect to a finite number of the independent variables from the set $\left\{x, u^{\lceil k\rceil}, z_{t}^{\langle-\ell\rangle}, k \geqslant 0, \ell \geqslant 1\right\}$ for the fixed time $t$ and are infinitely many times delta-differentiable with respect to $t$ if we set variables from the set $\left\{x, u^{\lceil k\rceil}, z_{t}^{\langle-\ell\rangle}, k \geqslant 0, \ell \geqslant 1\right\}$. Moreover, the operator $\Delta_{f}$ can be extended to $\mathscr{R}^{*}$ (we need this extension only when $t \neq 0$ ). If we extend operator $\Delta_{f}$ to variables $z_{t}^{\langle-i\rangle}, i \geqslant 1$, by using

$$
\Delta_{f}\left(z_{t}^{\langle-i\rangle}\right)=\frac{z_{t}^{\langle-i+1\rangle}-z_{t}^{\langle-i\rangle}}{t},
$$

then $\Delta_{f}\left(z_{t}^{\langle-i\rangle}\right) \in \mathscr{R}^{*}$ and the extension of $\Delta_{f}$ to $\mathscr{R}^{*}$ can be made by using the definition of operator $\Delta_{f}$.

Example 5 Suppose now that system (13) is defined on time scale $\mathbb{T}=\mathbb{Z}$, which corresponds to the standard discrete-time case. In this special case the graininess function $\mu$ is constant $(\mu=1)$, the time scale is homogeneous and the state transition map $x^{\sigma}=x+(x+u)^{2}$ does not depend on $\mu(t)$ (alternatively on $t$ ). One can choose (as in Example 4) $z_{t}:=u-\mu(t)(x+u)^{2}$. However, note that now the new variable $z_{t}$ does not depend on $t$, since $\mu(t)=1$ and we may define $z:=u-(x+u)^{2}$. Note also that when $\mathbb{T}=\mathbb{Z}$, all the points on $\mathbb{T}$ are isolated. Since there are no dense points on $\mathbb{Z}$, the new variables may be computed as in [1]. Moreover, note that the functions in the ring $\mathscr{R}$ do not depend on variable $t$ and so one can construct a quotient field of $\mathscr{R}$ as in [4].

If the system (13) is defined on time scale $\mathbb{T}=\mathbb{R}$, which corresponds to the continuous-time case, the graininess function $\mu=0$, the operator $\sigma_{f}=$ id (see Remark 6) and $\mathscr{R}^{*}=\mathscr{R}$.

\section{Conclusions}

In this paper the linear algebraic framework presented in [4] has been extended for nonlinear systems, defined on the nonhomogeneous, but regular time scale. This system description allows to unify the study of continuous and discrete-time systems, represented via difference operator. Moreover, it also covers the case of non-uniformly sampled systems. The developed framework can be applied to study different modeling, analysis and synthesis problems, including the different concepts of system equivalence and reduction, accessibility, realization, feedback linearization [1], model matching [15] etc.

Acknowledgments The work of Z. Bartosiewicz and M. Wyrwas is supported by the Białystok University of Technology grant No. S/WI/2/2011. The work of E. Pawłuszewicz was partly done during her stay in Aveiro University (Department of Mathematics). Her work is additionally supported by the Białystok University of Technology grant No. S/WM/2/08. The work of Ü. Kotta is supported by the Estonian Science Foundation Grant No. 6922. 
Open Access This article is distributed under the terms of the Creative Commons Attribution Noncommercial License which permits any noncommercial use, distribution, and reproduction in any medium, provided the original author(s) and source are credited.

\section{References}

1. Aranda-Bricaire E, Kotta Ü, Moog CH (1996) Linearization of discrete-time systems. SIAM J Control Optim 34(6):1999-2023

2. Atici FM, Biles DC, Lebedinsky AT (2006) An application of time scales to economics. Math Comput Model 43(7-8):718-726

3. Aulbach B, Hilger S (1990) Linear dynamic process with inhomogeneous time scale. In: Leonov G, Reitmann V, Timmermann W (eds) Nonlinear dynamics and quantum dynamical systems. Math Res, vol 59, pp 9-20

4. Bartosiewicz Z, Kotta Ü, Pawłuszewicz E, Wyrwas M (2007) Algebraic formalism of differential oneforms for nonlinear control systems on time scales. Proc Estonian Acad Sci Phys Math 56(3):264-282

5. Bartosiewicz Z, Kotta Ü, Pawłuszewicz E, Wyrwas M (2009) Differential rings associated with control systems on regular time scales. In: Proceedings of the European control conference 2009, Budapest, Hungary, pp 242-247

6. Bohner M, Peterson A (2001) Dynamic equations on time scales. Birkhäuser, Boston

7. Bohner M, Tisdell ChC (2005) Second order dynamic inclusions. J Nonlinear Math Phys 12(2):36-45

8. Cohn R (1965) Difference algebra. Wiley-Interscience, New York

9. Conte G, Moog C, Perdon A (2007) Algebraic methods for nonlinear control systems. Theory and applications, 2nd edn. Springer, London

10. Fliess M (1989) Automatique at corps différentiels. Forum Math 1:227-238

11. Glöckner H (2007) Finite order differentiability properties, fixed points and implicit functions over valued fields. arXiv:math/0511218

12. Gravagne IA, Davis JM, DaCunha JJ, Marks RJ (2004) Bandwidth reduction for controller area networks using adaptive sampling. In: Proceedings of international conference on robotic automation, New Orleans, LA

13. Grizzle J (1993) A linear algebraic framework for the analysis of discrete-time nonlinear systems. SIAM J Control Optim 31(4):1026-1044

14. Gürses M, Huseinov G, Silindir B (2005) Integrable equations on time scales. J Math Phys 46:113510$1-113510-22$

15. Moog CH, Perdon AM, Conte G (1991) Model matching and factorization for nonlinear systems: a structural approach. SIAM J Control Optim 29(4):769-785

16. Thomas D, Weedermann M, Billings L, Hoffacker J, Washington-Allen RA (2009) When to spray: a time-scale calculus approach to controlling the impact of west nile virus. Ecol Soc 14(2). http://www. ecologyandsociety.org/vol14/iss2/art21/ 